\title{
Effect of the Nuclear Factors EmBP1 and Viviparous1 on the Transcription of the Em Gene in HeLa Nuclear Extracts
}

\author{
Mona A. Razik and Ralph S. Quatrano' \\ Department of Biology and Curriculum in Genetics and Molecular Biology, University of North Carolina, Chapel Hill, \\ North Carolina 27599-3280
}

Templates constructed from the wheat $\mathrm{Em}$ and maize rab28 promoters are efficiently and accurately transcribed in the well-characterized cell-free transcription system prepared from HeLa nuclei. Deletion analysis of the Em promoter indicates that a G-box (CACGTG) element (Em1b) is required for transcription. USF, a Myc transcription factor in HeLa nuclear extracts, activates transcription by binding to Em1b, as shown by the ability of an antibody raised against USF to inhibit transcription and to interfere with Em1b complex formation in an electrophoretic mobility shift assay. The addition of the recombinant Viviparous 1 protein from maize to HeLa nuclear extracts specifically stimulated transcription of the Em promoter but was dependent on the presence of USF in the extract. In USF-depleted extracts, the addition of recombinant EmBP1, a basic leucine zipper transcription factor from wheat, activated transcription through Em1b as well as from a similar G-box in the adenovirus major late promoter. Our study demonstrates that the basic transcriptional apparatus in HeLa nuclear extract supports transcription from plant promoters and can be used to assay the function of certain plant nuclear proteins, thereby helping to determine their effects on transcription.

\section{INTRODUCTION}

The $E m$ gene encodes an abundant protein that is found in mature embryos of developing seeds of many plants. The phytohormone abscisic acid (ABA) and the regulatory locus viviparous1 (vp1) in maize (and its homolog in ABA-insensitive Arabidopsis [abi3]) are required for the embryo-specific expression of the Em gene (Marcotte et al., 1988; McCarty et al., 1991; Parcy et al., 1994; Rock and Quatrano, 1995). The $\mathrm{ABA}$ response element in the wheat $\mathrm{Em}$ promoter has been localized to a 76-bp segment (region 1) by a transient assay using rice protoplasts (Marcotte et al., 1988). Deletion of region 1, which contains two G-box (CACGTG) elements (Em1a and Em1b), eliminates the ABA response in transient assays, whereas mutations in either Em1a or Em1b dramatically reduce this $A B A$ response. Tetramers of either Em1a or Em1b in maize or rice protoplasts can confer ABA responsiveness to a nonresponsive cauliflower mosaic virus promoter (CaMV 35S) (Marcotte et al., 1989; Vasil et al., 1995).

Electrophoretic mobility shift assays have been used to identify the presence of DNA binding proteins in wheat and rice nuclear extracts that are specific for the G-boxes in region 1. A basic leucine zipper (bZIP)-type transcription factor, EmBP1, has been isolated by screening a wheat embryo cDNA library with an oligonucleotide probe containing region 1 (Guiltinan et al., 1990). Competition experiments using recombinant EmBP1 indicate that it preferentially binds

1 To whom correspondence should be addressed. E-mail rsq@unc. edu; fax 919-962-6840. to Em1a. In addition, a mutation in the core CACGTG of the Em1a element not only eliminates binding by nuclear extracts and EmBP1 but also eliminates the ABA response in transient assays (Guiltinan et al., 1990; Vasil et al., 1995). Consequently, EmBP1 has been identified as a factor that binds to Em1a and is thus implicated in the regulation of the Em promoter (Guiltinan et al., 1990).

EmBP1 belongs to a family of at least seven closely related DNA binding proteins in wheat (DeVos et al., 1991). More than $20 \mathrm{bZIP}$ proteins have been isolated from plant species, and all share the same core target sequence ACGT (Ehrlich et al., 1992; Katagiri et al., 1992; Kawata et al., 1992; Schindler et al., 1992a; Foley et al., 1993; Pysh et al., 1993; Zhang et al., 1993; Foster et al., 1994; Nantel and Quatrano, 1996). Of these bZIP proteins, a group has been identified that preferentially binds to the G-box sequence CACGTG. These are called G-box binding factors (GBFs) and include EmBP1 from wheat (Guiltinan et al., 1990), osZIP from rice (Nantel and Quatrano, 1996), TGA1a from tobacco (Katagiri et al., 1990), and the GBFs from Arabidopsis (Schindler et al.,1992b). These transcription factors have been shown to bind to the highly conserved G-box motif located on a variety of plant promoters that are inducible by such diverse signals as UV and red light (Giuliano et al., 1988; Weisshaar et al., 1991), drought, pathogen infections, and anaerobiosis regulation (Katagiri et al., 1989; Guiltinan et al., 1990; McKendree and Ferl, 1992). Schindler et al. (1992b) have shown that the proline-rich $\mathrm{N}$-terminal domain of Arabidopsis 
GBF1, when fused to the yeast GAL4 binding domain, can stimulate transcription in plant protoplasts and mouse 3T3 cells.

Studies using ABA-insensitive mutants have shown that the regulatory locus $v p 1$ is required for $E m$ expression (McCarty et al., 1991). In addition, several reports (McCarty et al., 1991; Hattori et al., 1994; Vasil et al., 1995; Hill et al., 1996) have demonstrated that overexpression of the VP1 protein in maize or rice protoplasts can transactivate the $E m$ promoter at subthreshold levels of endogenous $A B A$. In the presence of exogenous $A B A$ and overexpression of VP1 in these protoplasts, a synergistic activation of $E m$ expression has been observed. The region in the $E m$ promoter required for VP1 transactivation and the synergistic effect with ABA overlaps with region 1 (Hattori et al., 1995; Vasil et al., 1995). Similar to the ABA response, tetramers of either Em1a or Em1b were shown to be sufficient for the VP1 responses, whereas mutations in the G-boxes within region 1 of the Em promoter prevented VP1-mediated expression (Marcotte et al., 1989; Vasil et al., 1995). Hence, promoter elements required for $\mathrm{ABA}$ and VP1 to enhance expression of the $E m$ gene reside within region 1 and correspond to the G-box(es).

Analyses of the VP1 sequence and functional assays indicate that the N-terminal domain of VP1 can serve as a transcriptional activator (McCarty et al., 1991). Electrophoretic mobility shift assays also indicate that a truncated VP1 without activation can enhance the binding of a variety of transcription factors to their target sequences (Hill et al., 1996), including EmBP1 to Em1a and Em1b. This nonspecific enhancement effect of VP1 in vitro may occur through a weak, nonspecific, and transient association of VP1 with DNA. However, the ability of VP1 to enhance the binding of sequence-specific transcription factors in vitro has not been correlated with its effect in transactivating the Em promoter in protoplasts and transgenic plants. Recently, Suzuki et al. (1997) showed that the conserved B3 domain of VP1 can specifically bind to the Sph element in the $C 1$ gene from maize. However, unlike the regulation of $\mathrm{C1}$, neither the Sph element in the Em promoter nor the B3 domain of VP1 is required for $E m$ expression (Hill et al., 1996).

One approach to determining the mechanism(s) by which EmBP1 and VP1 exert their effects on Em expression is to use a well-characterized cell-free transcription system. For example, can recombinant EmBP1 activate transcription in vitro and can VP1 enhance transcription through a sequence-specific factor as a coactivator? Some success has recently been reported for plant in vitro transcription systems (Yamagauchi et al., 1994; Sugiura, 1996). A wheat germ chromatin extract was developed that accurately transcribed from the initiator sequence in the CaMV 35 S minima promoter and was stimulated by the DNA binding protein TGA1a (Yamazaki et al., 1990; Schweizer and Mozinger, 1994). Nuclear extracts from parsley (Frohnmeyer et al., 1994) and tobacco cells (Fan and Sugiura, 1994) have also been shown to support cell-free transcription. Most recently, a whole-cell extract from rice suspension cultures was reported to be transcriptionally competent and dependent on the TATA box, the initiator sequence, and specific spacing requirements between the initiator and the TATA box for accurate transcription. However, none of these plant systems has been used to analyze functional interactions between cis elements and added plant transcription factors, nor have they been reproducibly used with a variety of plant promoters (Zhu et al., 1995).

The basic transcription machinery appears to be highly conserved between plants and animals and may share a common mechanism of transcriptional activation (Haas and Feix, 1992; Mukumoto et al., 1993). For example, the yeast acidic transeription factor GAL4 was shown to activate transcription in plant (Ma et al., 1988), Drosophila (Fischer et al., 1988), and mammalian (Kakidani and Ptashne, 1988) cells. In fact, the plant transcription factor TGA1a from tobacco was shown to activate transcription from the CaMV 355 promoter in a human reconstituted in vitro transcription system (Katagiri et al., 1990). Given these results, we decided to test the ability of the well-characterized and widely used HeLa nuclear extract (HNE) not only to support transcription from the plant $E m$ promoter but also to determine how the plant factors EmBP1 and VP1 might function with the basic transcriptional apparatus of HeLa cells.

We report that templates containing G-boxes from the wheat $E m$ and maize rab28 genes (Pla et al., 1993) are accurately and efficiently transcribed in the HNE. The conserved $\mathrm{G}$-box element in the $\mathrm{E} m$ promoter is required and apparently is recognized by the mammalian Myc transcription factor USF, which is present in the HNE. VP1 without its transcriptional activation domain stimulated transcription in the HNE by using the Em template but was dependent on the presence of USF. We show that recombinant EmBP1 can activate not only the $E m$ promoter but also the adenovirus major late $(A d M L)$ viral promoter in the HNE depleted of USF. These results indicate that the HNE can be used to accurately transcribe plant genes that contain cis elements that are recognized by HeLa factors. Furthermore, HNE can be used to test the effect of certain plant nuclear factors on transcription.

\section{RESULTS}

\section{Transcription from the Em and the rab28 Promoters Using the HNE}

The $113 \mathrm{kp}$ plasmid, containing 642 bases of the Em promoter fused to a $\beta$-glucuronidase (GUS) reporter gene (Em113kp), was linearized with EcoRV and used as a template to generate run-off transcripts in the HNE. One major transcript (697 nucleotides) of the correct size (Figure 1A) and one minor transcript were detected in the in vitro transcription assay (Figure $2 \mathrm{~A}$, lane 2). The addition of RNase $A$ 
A

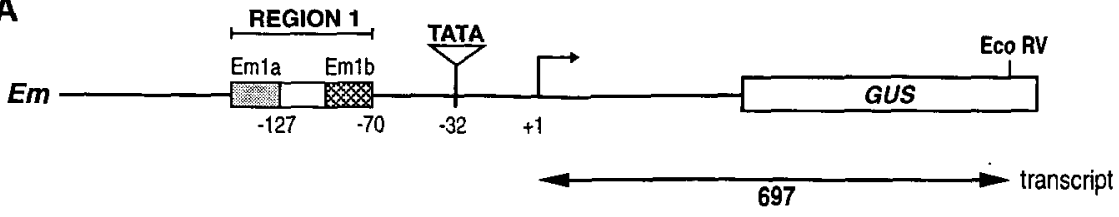

B

$$
\begin{aligned}
& \text { Em1a CGGACACGIGGCG } \\
& \text { Em1b TGCACACGTGCCG } \\
& \text { rab28 C G C - CACGTGG G C } \\
& A d M L G G C-C A C G T G A C C
\end{aligned}
$$

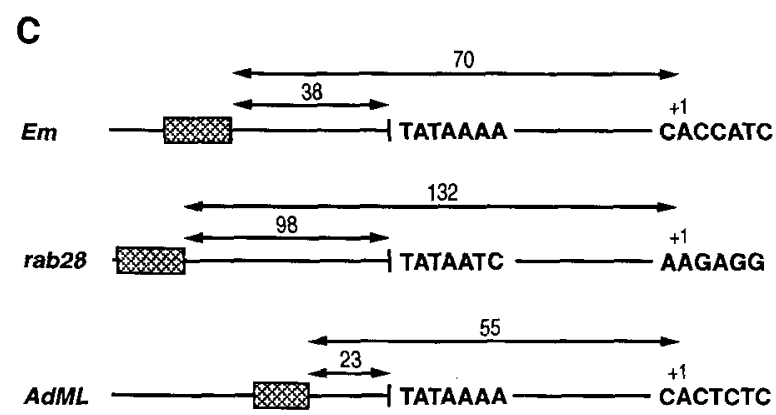

Figure 1. Diagram of Various Promoters and G-Box Sequences Used in This Study.

(A) Map of the Em promoter-GUS template and the position and length (in nucleotides) of the main transcript produced in the HNE.

(B) Comparison of the regions in the Em (Em1a and Em1b), rab28, and the AdML promoters containing G-boxes (underlined and boldface letters).

(C) Comparison of the Em, rab28, and the AdML promoters with respect to the distances (in bases) between the transcription initiation site ( +1 ), TATA, and the G-box (crosshatched) closest to the TATA box.

to the transcription reaction digested the products (Figure $2 \mathrm{~B}$, lane 2 ), whereas incubation with $\alpha$-amanitin inhibited accumulation of the products (Figure $2 \mathrm{~B}$, lane 3 ). The rab28 promoter fused to the GUS reporter gene was used as another plant promoter similar to Em (Pla et al., 1993; see Figures $1 \mathrm{~B}$ and $1 \mathrm{C}$ ). The rab28 plasmid was also digested with EcoRV and used as a template to generate run-off transcripts in the HNE. Lane 4 of Figure $2 \mathrm{C}$ shows that the rab28 template generated a single transcript of the correct size (650 nucleotides). The addition of RNase A to the transcription reaction digested the products (Figure $2 \mathrm{C}$, lane 2 ), whereas incubation with $\alpha$-amanitin inhibited the accumulation of the products (Figure $2 \mathrm{C}$, lane 3 ). These results indicate that the RNA products detected from the Em and rab28 promoters in the HNE were RNA transcripts generated by RNA polymerase II activity.

The $5^{\prime}$ start site of the main Em transcript (CCACC) was mapped on the Em promoter by a primer extension assay and found to be 32 bases downstream from the TATA box (Figure 3), which corresponds to the in vivo start site and the observed 697-nucleotide transcript (Figures 1A and 2A). The start site of the minor transcript is $5^{\prime}$ to the start of the major transcript but was not mapped.

\section{Em1b, a G-Box Element in the Em Promoter, Is Sufficient for Transcription in the HNE}

Deletions in the $5^{\prime}$ regions of the Em113kp (Figure 4A) were used as templates to determine the regions required for transcription in the HNE. Transcription levels of templates 1 to 4 were equivalent, as shown in Figure $4 B$ (lanes 2 to 5). However, without the region between nucleotides -57 to -38 (template 5), no transcripts were detected (Figure 4B, lane 6). Competition with an excess of the Em1b oligonucleotide inhibited transcription from template 4 (Figure 4C, 
A

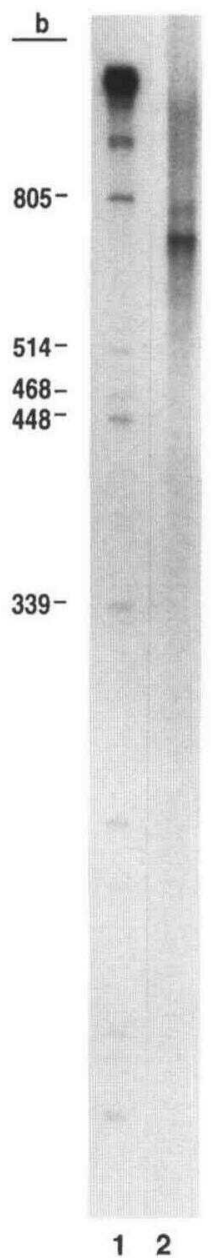

B

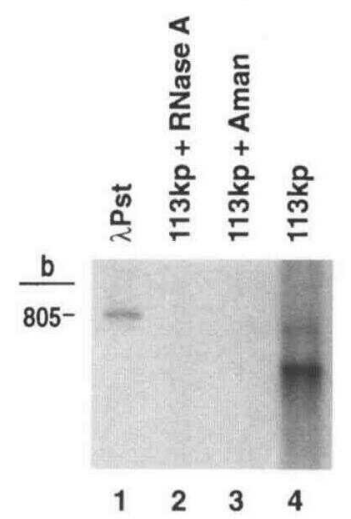

C
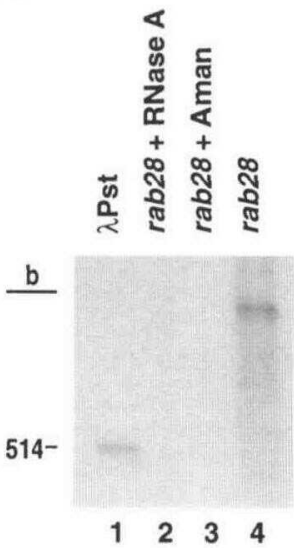

Figure 2. Transcription Reactions in the HNE Using the Em and the rab28 Promoters.

(A) Lane 1 contains a $\lambda$ Pstl digest, with the base (b) size markers indicated at left. Lane 2 contains the transcripts generated from the Em promoter-GUS template (Em113kp; see Figure 4A).

(B) Lane 1 contains a $\lambda$ Pstl ( $\lambda$ Pst) marker ( 805 bases). Lanes 2 to 4 contain transcription reactions with the Em113kp (113kp) template and the HNE (100 to $150 \mu \mathrm{g}$ of protein). The reaction in lane 2 had been treated with $2 \mu \mathrm{g}$ of RNase A. The reaction in lane 3 included 1 $\mu \mathrm{g} / \mathrm{mL} \alpha$-amanitin (Aman). The two $E m$ transcripts from the Em113kp template are visible in lane 4 only. The main transcript is 697 nucleotides long (see Figure 1A).

(C) Lane 1 contains a $\lambda$ Pstl marker (514 bases). Lanes 2 to 4 contain transcription reactions with the rab28 template and the HNE (100 to $150 \mu \mathrm{g}$ of protein). The reaction in lane 2 had been treated with $2 \mu \mathrm{g}$ of RNase A. The reaction in lane 3 included $1 \mu \mathrm{g} / \mathrm{mL} \alpha$-amanitin. A single transcript from the rab28 template is visible in lane 4 only (650 nucleotides long).

lanes 2 and 3) compared with competition with the mutant Em1b oligonucleotide that lacks the CACGTG core sequence (Figure $4 \mathrm{C}$, lanes 4 and 5). The Em1b oligonucleotide, when added to the transcription assay at the same molar excess, was a more effective inhibitor of transcription from template 4 than was the mutant Em1b oligonucleotide (in Figure 4C, compare lane 2 with lane 4 and lane 3 with lane 5). These results indicate that a single G-box closest to the TATA sequence (Figure 1A, Em1b) appeared to be sufficient for transcription in the HNE.

Experiments were also performed with the HNE to compare transcription rates from the $E m$ promoter with two other promoters active in plants, that is, ubiquitin from maize (Christiansen et al., 1992) and CaMV 35S (Marcotte et al., 1989). There were no visible transcripts from the ubiquitin or the CaMV $35 \mathrm{~S}$ promoters in the HNE when the conditions and exposure time were the same as those used for Em113kp (Figure 4B, lanes 7 and 8). Both the ubiquitin and CaMV $35 S$ promoters lacked G-box sequences, although the CaMV 35 S has a related Hex-1 sequence (TGACG), which is apparently responsible for the transcripts generated from the CaMV 35S promoter in the HNE, as reported by Katagiri

\section{A C G T}

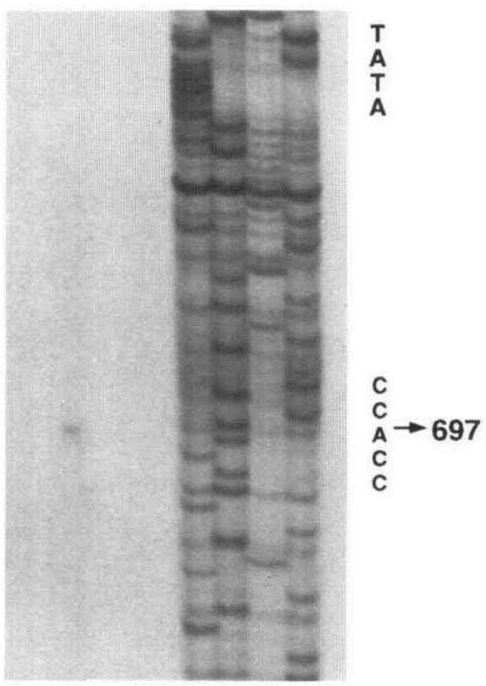

Figure 3. Primer Extension Analysis of the Major Em Transcript Generated in the HNE.

The RNA synthesized in an in vitro transcription assay was analyzed by primer extension. Deoxynucleotide sequencing reactions of the Em promoter and primer extension products were fractionated on an $8 \%$ denaturing polyacrylamide gel. DNA sequences immediately adjacent to the RNA start site (arrow) of the Em promoter are shown. The single major transcript (697 nucleotides) can be seen to the left of the sequencing lanes. The primer used was 5 '-GGTGCTCGACCGCTAAGGTT-3'. 
A

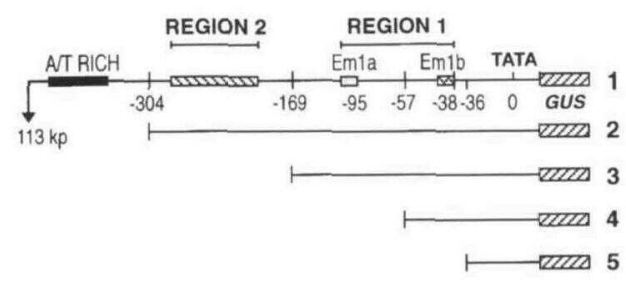

B

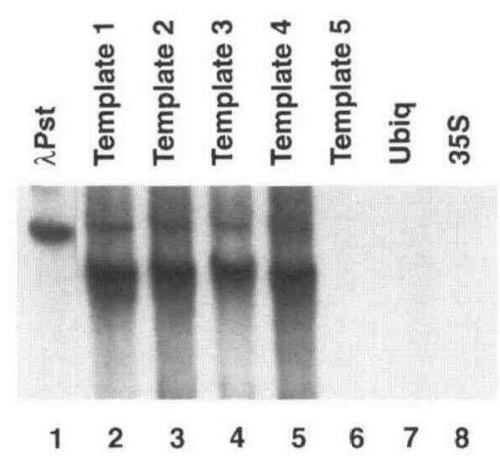

C

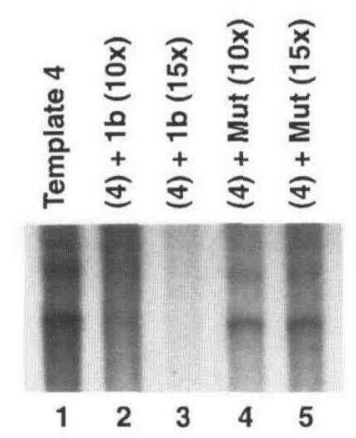

Figure 4. Region of the Em Promoter Required for Transcriptional Activity in the HNE.

(A) Full map of Em113kp and the deletions used as templates in the transcription reactions. Template 1 (1) contains the entire Em113kp construct; template 2 (2) lacks the AT-rich region. Template 3 (3) contains only region 1; template 4 (4) has only the Em1b G-box. Template 5 (5) lacks a G-box but has all elements of the Em promoter 3' of Em1b.

(B) Transcripts generated in the HNE from the templates shown in (A). Lane 1 contains a $\lambda$ Pstl ( $\lambda$ Pst) marker ( 805 bases). Lanes 2 to 6 contain transcription reactions generated from templates 1 to 5 , respectively. Lanes 7 and 8 contain transcription reactions generated using the ubiquitin (Ubiq) or the CaMV 35S (35S) promoter template, respectively. (C) Transcription reactions using template 4 (4) in the HNE. Lane 1 is the control transcription reaction; lanes 2 and 3 contain transcription reactions in the presence of Em1b oligonucleotide competitor at 10fold $(10 x)$ and 15 -fold $(15 x)$ the molar concentration of template 4 , respectively. Lanes 4 and 5 contain transcription reactions in the presence of a mutant (Mut) Em1b oligonucleotide competitor at 10fold and 15 -fold the molar concentration of template 4 , respectively. et al. (1990). However, the rate of transcription in the HNE of the CaMV $35 \mathrm{~S}$ promoter was significantly less than that of the Em113kp under our conditions.

\section{USF Appears to Be the Protein in the HNE That Binds to the Em1b Element}

Electrophoretic mobility shift assays in the presence of saturating concentrations of poly(dl- $d C)$ revealed the presence of a protein(s) in the HNE that specifically bound to the Em1b probe (Figure 5A, lane 4) and was used in a competition assay with an excess of the Em1b oligonucleotide (Figure $5 \mathrm{~A}$, lanes 5,7 , and 9). This protein(s) in the HNE was not competed with equivalent concentrations of the mutant Em1b oligonucleotide, as seen in lanes 6, 8, and 10 (Figure $5 A)$. This was further indication that the HeLa protein(s) is specific to the G-box element Em1b.

The addition of the USF antibody (anti-USF) to this electrophoretic mobility shift assay resulted in a dose-dependent disappearance of the retarded band and a supershift to a slower moving position on the gel (Figure 5B, lanes 12 and 13). Preimmune sera had no effect (Figure $5 B$, lane 14). The effect of anti-USF on the HNE also influenced transcription. This effect on transcription by Em113kp (Figure 6, lane 2) was specific because the anti-USF had little effect on transcription when a template from the dihydrofolate reductase (DHFR) promoter lacking a G-box was used (Farnham and Means, 1990) (in Figure 6, compare lanes 1 and 2 with lanes 3 and 4). The antibody raised against the plant bZIP transcription factor EmBP1, which recognizes G-box elements in the Em promoter (Guiltinan et al., 1990), had no effect on transcription in the HNE (data not shown).

\section{Recombinant EmBP1, VP1, and USF Stimulate Transcription Using the Em Promoter in the HNE}

When the recombinant EmBP1 fusion protein was added to the HNE with Em113kp (Figure 4A, template 1), only the main transcript from the +1 start site (Figure $1 \mathrm{~A}$ ) was detected (Figure 7A, lane 3). This effect of EmBP1 is specific to the Em promoter because transcription with the DHFR promoter (which also has multiple transcription start sites) was unaffected by the addition of EmBP1 (Figure 7B, lanes 3 and 4). When the truncated VP1 fusion protein (lacking the transcriptional activation domain) or the purified USF protein was added to the HNE, transcription from the Em promoter was stimulated to approximately the same levels (Figure $7 \mathrm{C}$ ). However, unlike the effect of EmBP1, both transcripts from Em113kp were detected when either VP1 or USF was added to the HNE. Stimulation of transcription using VP1 appeared to be specific to the Em promoter because no stimulation was observed when the DHFR promoter was used (Figure 7B, lane 5). A similar specificity 
A

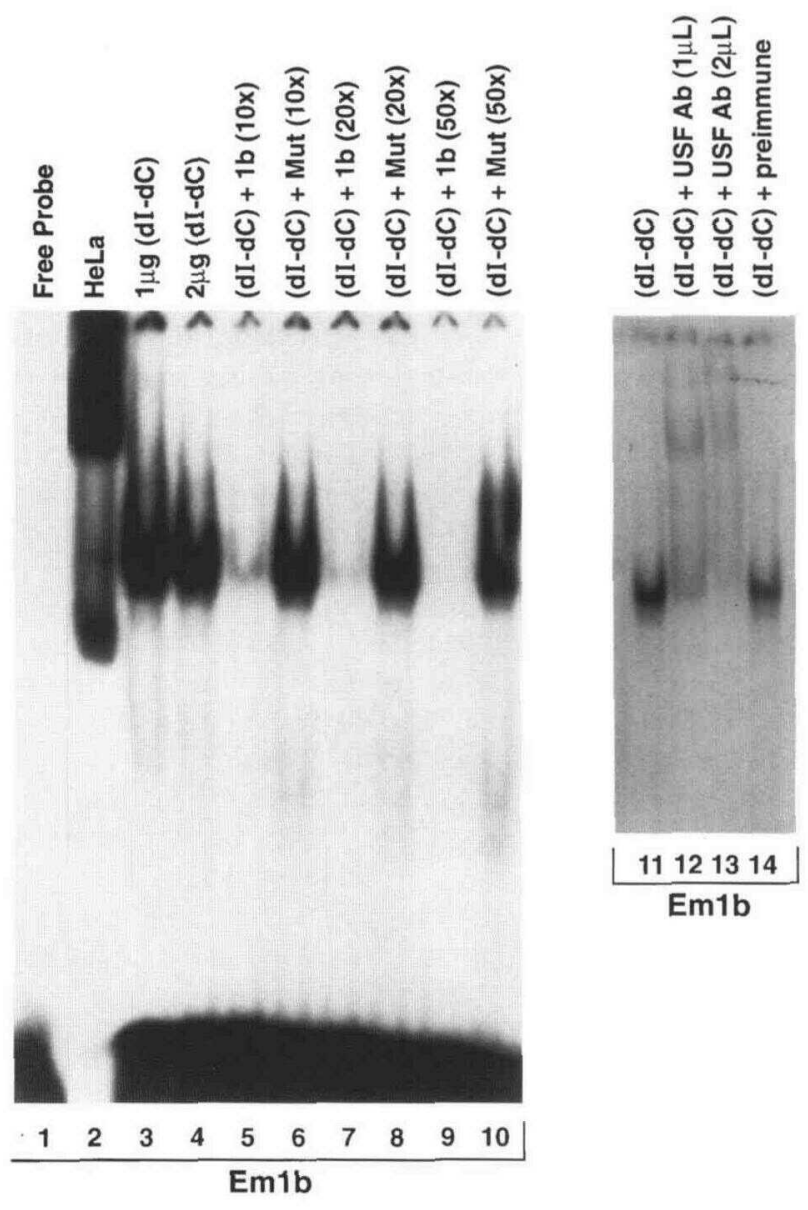

Figure 5. Electrophoretic Mobility Shift Assays Using the HNE and Em1b as Probe.

(A) Electrophoretic mobility shift assays in the absence (lane 1) and presence (lane 2) of the HNE and with the HNE in the presence of 1 $\mu \mathrm{g}$ (lane 3) or $2 \mu \mathrm{g}$ (lanes 4 to 10 ) of poly(dl-dC) ([dl-dC]). A 10-fold (10X), 20 -fold $(20 \times)$, and 50-fold (50X) molar excess of cold Em1b oligonucleotide competitor was included in the gel shift assays in lanes 5, 7, and 9, respectively. A 10-, 20-, and 50-fold molar excess of a mutant (Mut) Em1b oligonucleotide was included in the gel shift assays in lanes 6, 8, and 10, respectively. Each reaction contains 5 $\mu \mathrm{g}$ of the HNE protein and $1 \mathrm{ng}$ of the labeled Em1 $\mathrm{b}$ probe.

(B) Electrophoretic mobility shift assays in the presence of the USF antibody. Lanes 11 to 14 are identical to lane 4 in (A) except that lanes 12 and 13 contain 1 and $2 \mu \mathrm{L}$ of the anti-USF (USF Ab), respectively, and lane 14 contains the preimmune control. The antiUSF and the preimmune control were preincubated with the HNE for $10 \mathrm{~min}$ before the addition of the labeled Em1b probe. for the Em promoter was demonstrated when USF was used (data not shown).

\section{AdML and Em Promoters Are Recognized by EmBP1 in the HNE Immunodepleted of USF}

Different-sized transcripts were generated from the AdML and $E m$ promoters in the HNE (Figure $8 \mathrm{~A}$ ). Transcription from Em113kp was drastically reduced in HNE immunodepleted of USF (Figure 8B, lane 2). When full-length recombinant EmBP1 was added to the immunodepleted HNE, we observed not only transcription from the main transcriptional start site but also stimulation of transcription (Figure $8 \mathrm{~B}$, lane 3). We have reproducibly observed a two- to threefold enhancement of the 697-nucleotide transcript when compared with the combined expression levels of the major and minor transcripts in the HNE. Truncated recombinant VP1 had no effect on transcription in immunodepleted HNE, either alone (Figure 8D, lane 3) or with EmBP1 or USF (data not shown).

The AdML promoter contained a single CACGTG site 23 bp upstream of the TATA sequence and was very similar in sequence and position to the rab28 promoter and the Em1b element in the $\mathrm{Em}$ promoter (Figures $1 \mathrm{~B}$ and $1 \mathrm{C}$ ). When whole $\mathrm{HNE}$ was used to transcribe the AdML promoter, there was no detectable effect when EmBP1 was added (data not shown). However, when the HNE depleted of USF was used with AdML, the effects of EmBP1 were similar to that previously observed with the Em promoter, that is, a two- to threefold stimulation of transcription (Figure $8 \mathrm{C}$, lane

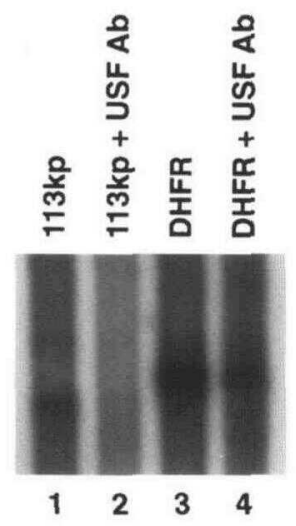

Figure 6. Effects of the USF Antibody on Transcription from the Em and DHFR Promoter Templates in the HNE.

Control transcription reactions contain either the Em113kp (113kp) template (lane 1) or the DHFR template (lane 3). Transcription reactions preincubated with anti-USF (USF Ab) $(1 \mu \mathrm{L}$ of anti-USF/15 $\mu \mathrm{L}$ of the HNE) are shown in lane 2 (Em113kp template) and lane 4 (DHFR template). 

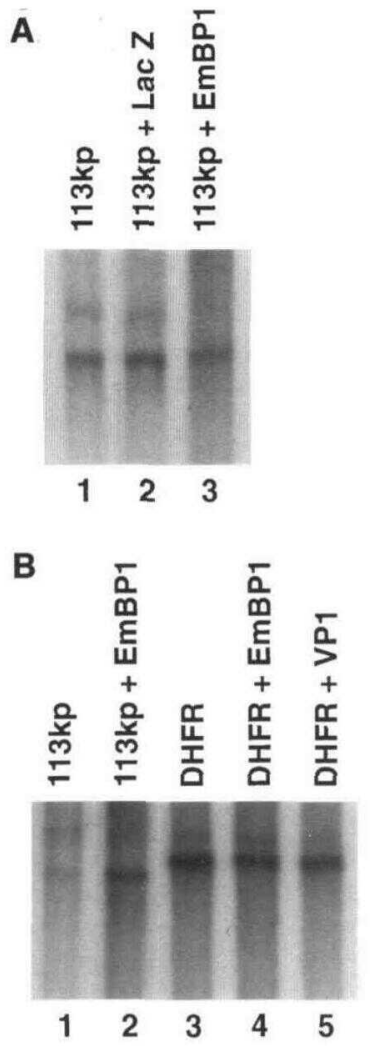

C

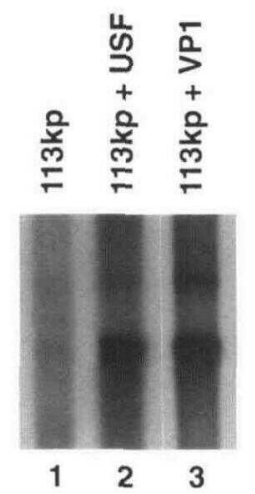

Figure 7. Effects of the Recombinant Maltose-Binding Fusion Proteins EmBP1 and VP1 on Transcription from the Em and DHFR Promoter Templates in the HNE.

(A) Transcription reactions containing the Em113kp (113kp) template (lane 1) in the presence of $0.5 \mu \mathrm{g}$ of LacZ (lane 2) or $0.5 \mu \mathrm{g}$ of EmBP1 (lane 3).

(B) Transcription reactions containing the Em113kp (lanes 1 and 2) or the DHFR (lanes 3 to 5) templates in the presence of $0.5 \mu \mathrm{g}$ of EmBP1 (lanes 2 and 4) or $0.6 \mu \mathrm{g}$ of VP1 (lane 5).

(C) Transcription reactions containing the Em113kp template (lane 1) in the presence of $25 \mathrm{ng}$ of recombinant USF (lane 2) or $0.6 \mu \mathrm{g}$ of VP1 (lane 3).
3). Therefore, it appears that USF and EmBP1 can interchangeably stimulate transcription from either the $E m$ or AdML promoter through their respective CACGTG core elements.

\section{DISCUSSION}

\section{Transcription Reaction}

We report here the detection of RNA transcripts in the HNE by using templates composed of the wheat $E m$ and maize rab28 promoters fused to the GUS reporter gene as well as the $A d M L$ promoter. We have shown that transcription from the $\mathrm{Em}$ template resulted in a major transcript of 697 nucleotides that starts 32 bases downstream of the TATA box. The initiation site of the main transcript $(+1)$ from the $E m$ promoter in the HNE is CCACCATC, which is similar to that of the AdML promoter (CACTCTC). Both are in agreement with the consensus (CAYYYTC) reported by Roy et al. (1991) and Kozak (1984). Studies of other plant transcription start sites (Joshi, 1987; Zhu et al., 1995) and the prediction by Litts et al. (1991) for the Em initiation site confirm that CCACCATC is the correct site for the Em gene. We also have evidence that the site of the main transcript identified in the HNE is the likely start site in vivo. When we performed primer extension assays, one of the bands generated from wheat poly $(A)^{+}$RNA was identical in size to the major transcript from the HNE. Because we did not detect the minor size transcript in vivo, we believe that the larger minor transcript generated in the HNE is likely to be an artifact of in vitro transcription.

Although the HNE transcription system has been used previously to express two polymerase II genes from plants (Boston and Larkins, 1986; Katagiri et al., 1990), this study shows that both the Em and the rab28 promoters are transcribed at much higher levels than either of the previously used promoters. We believe this is due primarily to the presence of a CACGTG target sequence (i.e., G-box) in both promoters (Litts et al., 1991; Pla et al., 1993). These same G-box elements in the Em promoter (i.e., Em1a and Em1b) have been shown to be responsive to both the ABA and VP1 signals to activate $E m$ expression in transient assays (Vasil et al., 1995). Furthermore, the G-boxes in the rab28 and Em promoters are identical to the binding site for USF (Figure 1), the endogenous transcriptional activator in the HNE, and the sequence in the AdML promoter that is recognized by USF (Sawadogo and Roeder, 1985). In addition, the flanking sequences on either side of the G-boxes in these promoters are conserved, for example, nine of 13 bases are the same in the Em1b and AdML elements (Figure 1B). The positions of the G-boxes relative to the TATA box are also very similar; in the AdML promoter, the G-box is positioned 23 bases upstream of the TATA, whereas in the Em promoter, Em1b is 38 bases upstream of the TATA sequence. Finally, these two 
A

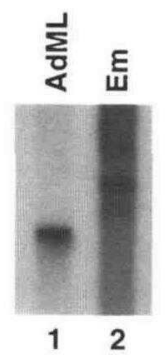

C

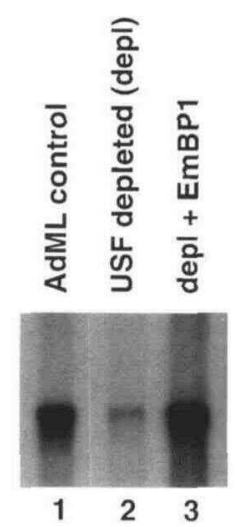

B



D

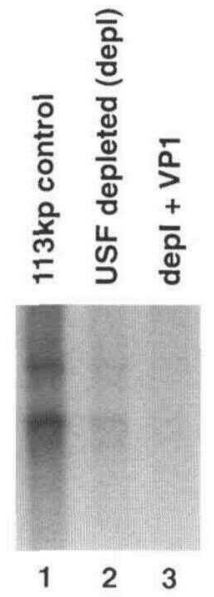

Figure 8. Effect of the Recombinant Maltose-Binding Fusion Protein EmBP1 on Transcription from the Em Promoter and the AdML Promoter Templates.

(A) Transcription reactions contain the AdML (lane 1) and the Em113kp (Em; lane 2) templates in the HNE.

(B) Lane 1 is the transcription reaction containing the Em113kp (113kp) template and HNE that was passed over a protein A-Sepharose column. A transcription reaction containing the Em113kp template and the HNE was passed over an anti-USF protein A-Sepharose column (immunodepleted [depl] of USF) (lanes 2 and 3 ) in the absence (lane 2) or presence (lane 3) of $0.5 \mu \mathrm{g} \mathrm{EmBP1.}$

(C) Lane 1 is the transcription reaction containing the AdML template and the HNE that was passed over a protein A-Sepharose column. A transcription reaction containing the $A d M L$ template and the HNE was immunodepleted of USF (lanes 2 and 3 ) in the absence (lane 2) or presence (lane 3) of $0.5 \mu \mathrm{g}$ EmBP1.

(D) Lane 1 is the transcription reaction containing the Em113kp template and the HNE that was passed over a protein A-Sepharose column. A transcription reaction containing the Em113kp template and the HNE was immunodepleted of USF (lanes 2 and 3 ) in the absence (lane 2) or presence (lane 3) of 0.6 $\mu \mathrm{g}$ VP1.

promoters have identical TATA boxes (TATAAAA), which are very strong TATA binding protein sequences (Mukumoto et al., 1993), and similar initiators; the initiator is 55 bases from the G-box (CACTCTC) in AdML and 70 bases from the G-box Em1b (CACCATC) in the Em promoter (Figure 1C). Hence, the similarity between the response elements of these plant promoters and those recognized by endogenous factors in the HNE contributes to the high levels of expression from these plant templates.

Additional data support the conclusion that the USF protein in the HNE is the factor responsible for transcription from the Em promoter through the Em1b element. Using 5 deletions of the Em promoter, we found that Em1b is essential for transcription of the Em promoter in the HNE. Competition assays performed with an excess of the Em1b oligonucleotide inhibited transcription when the Em promoter was used and interfered with gel mobility shift assays when the HNE was used. Both of these results are similar to those obtained with other eukaryotic G-box-containing promoters that require USF, such as the U6 gene in sea urchin (Li et al., 1994) and AdML (Sawadogo and Roeder, 1985). The addition of anti-USF to the gel retardation assay using the $\mathrm{Em}$ template resulted in a dose-dependent disappearance of the retarded band and a supershift at a higher position on the gel. Furthermore, we show that when USF was immunodepleted from the HNE, transcription from the Em or AdML templates was reduced dramatically. To determine whether the HNE could be used to asses the function of certain factors believed to interact with the $\mathrm{Em}$ promoter, we added recombinant EmBP1 and VP1 proteins to transcription reactions containing the $E m$ template.

\section{Effects of VP1 on Transcription}

VP1 is required for Em expression (McCarty et al., 1991; Hattori et al., 1994; Vasil et al., 1995; Hill et al., 1996). Functional assays indicate that the $\mathrm{N}$-terminal region (120 amino acids) of VP1 is a transcriptional activator (McCarty et al., 1991), but we have been unable to detect specific binding of VP1 to DNA (Hill et al., 1996). Our results indicate that a truncated VP1 (lacking an activation domain) dramatically enhances the binding of a variety of transcription factors to their target sequences, including EmBP1 to region 1 of the Em promoter (Hill et al., 1996). This in vitro enhancement effect of VP1 appears to be nonspecific and may occur through a weak and transient association of a highly conserved region of VP1 (BR2) with DNA (Hill et al., 1996). However, it is not clear from the work of Hill et al. (1996) whether the enhancement effect of VP1 in vitro is related to its ability in transient assays (Vasil et al., 1995) and in transgenic plants (Parcy et al., 1994) to activate transcription from the Em promoter.

In this study, our results indicate that when VP1 is added to the whole HNE, transcription from the Em template is stimulated. Purified USF also stimulated transcription, but 
$\sim 20$ times more VP1 was needed to achieve the same level of transcript. The most likely explanation is that the truncated VP1 lacked its transcriptional activation domain and as such affected transcription by interacting with endogenous transcription factors, whereas USF was the endogenous, sequence-specific transcription factor that affected transcription directly. We believe that VP1 enhancement of transcription requires not only USF but other essential components found in whole HNE, because in immunodepleted HNE, VP1 had no effect, either alone or when used in combination with EmBP1 or USF. These conclusions are consistent with our in vitro results showing that VP1 cannot bind DNA alone but rather facilitates the binding of a wide range of sequence-specific factors to their target sites, either directly or through interactions with other proteins localized to the transcriptional complex.

Our view that VP1 appears to be a partner of USF is similar to the conclusions of Timchenko et al. (1995), who showed that the C/EBP protein can autoregulate its own gene by enhancing the binding of USF to a G-box present on the C/EBP promoter. C/EBP does not bind to its own promoter, but its presence presumably stimulates transcription by enhancing the binding of USF to its target G-box. It is possible that VP1 affects USF in a similar manner, that is, by stimulating transcription as a coactivator. This would make it possible for VP1 to coactivate transcription through different transcription factors without being required for basal transcription (Chen et al., 1994). By VP1 interacting with a variety of transcription factors to activate a wide range of different genes in diverse metabolic and developmental pathways is consistent with its pleiotropic effects of VP1 in maize and its homolog abi3 in Arabidopsis (see Hill et al., 1996). However, the effects of VP1 on expression of embryonic maturation genes are also very specific. For example, both the rab28 and the catalase cat1 genes in maize are regulated similar to $\mathrm{Em}$, that is, they are responsive to $\mathrm{ABA}$ and are temporally expressed at the same time during embryo maturation, but unlike Em, neither rab28 nor cat1 requires VP1 for expression (Pla et al., 1991; Williamson and Scandalios, 1992). A model in which VP1 functions as a coactivator capable of activating specific genes with different DNA binding partners may achieve the required level of specificity.

\section{Effects of EmBP1 on Transcription}

Because activation of the $E m$ promoter is significantly reduced in USF-depleted extracts, we used this extract to determine whether recombinant EmBP1 can stimulate transcription from the Em template. We found that when EmBP1 is added to the HNE depleted of USF, transcripts from both the $E m$ and $A d M L$ templates accumulate. This provides evidence that EmBP1 can directly activate transcription from a G-box in the Em promoter. In addition, EmBP1 directs transcription from the in vivo start site only, resulting in a single 697-nucleotide transcript; no additional transcripts were de- tected when EmBP1 was added to either the immunodepleted HNE or whole HNE. The effect of EmBP1 directing transcription from one site on the $\mathrm{Em}$ promoter can be compared to the effect of Sp1 and E2F directing transcription on the DHFR promoter in the HNE. Each of these cell cycleregulated transcription factors directs transcription from a different start site in the DHFR promoter; the $\mathrm{Sp} 1$ site is used for basal transcription, whereas the E2F site is required for growth (Schilling and Farnham, 1995). Therefore, by using USF-depleted extracts of the HNE, one should be able to test the activation function of purified transcription factors that recognize CACGTG target sites as well as their interaction with the conserved basal transcriptional machinery found in the HNE. However, although VP1 stimulated transcription from whole HNE, we did not observe the same effect when we added purified USF (or EmBP1) in combination with VP1 to the USF-depleted extracts. Perhaps, components essential for the VP1 enhancement were removed in the preparation of USF-depleted extract.

In summary, use of the HNE to activate transcription from plant promoters will allow investigators to analyze a promoter structurally and study the effects of transacting factors on their target sites. It might also be possible to study transcription from plant or other promoters that have low levels of expression in the HNE (e.g., zein and CaMV 35S) by enhancing their basal transcription rate to detectable levels by introducing G-box sequences into the given template. Futhermore, we may determine those factors required for the specificity of VP1 action. We observed specificity in the VP1 enhancement of transcription in the HNE; that is, VP1 could discriminate between the $E m$ template and USF (which resulted in enhanced transcription rates) and the DHFR template and its sequence-specific factor in the HNE (which resulted in no enhanced transcription rates). Finally, our results with recombinant EmBP1 and VP1 in HNE suggest that one can identify functional domains in EmBP1 and VP1 that specifically initiate and enhance transcription from a given template. In addition, using USF-depleted extracts as an assay, we may be able to identify and characterize components in whole HNE (or plant extracts) that support the specificity of VP1 enhancement.

\section{METHODS}

\section{Preparation of the HeLa Nuclear Extract}

The HeLa nuclear extract (HNE) was prepared from frozen HeLa nuclei (Cellex Biosciences Inc., Minneapolis, MN), according to the method of Dignam et al. (1983). The nuclei were thawed and extracted in buffer $\mathrm{C}(20 \mathrm{mM}$ Hepes, $\mathrm{pH} 7.9,0.2 \mathrm{mM}$ EGTA, $2 \mathrm{mM}$ DTT, $25 \%$ glycerol, $0.15 \mathrm{mM}$ spermine, $0.75 \mathrm{mM}$ spermidine, 1.0 $\mathrm{mM}$ phenylmethylsulfonyl fluoride, and $0.4 \mathrm{M} \mathrm{NaCl})$. The extract was recovered by centrifugation at $49,000 \mathrm{rpm}$ for $45 \mathrm{~min}$ and then dialyzed for $2 \mathrm{hr}$ against buffer D (20 mM Hepes, $\mathrm{pH} 7.9,20 \%$ glycerol, $100 \mathrm{mM} \mathrm{KCl}, 0.2 \mathrm{mM}$ EDTA, $0.2 \mathrm{mM}$ EGTA, $2 \mathrm{mM}$ DTT, $1.0 \mathrm{mM}$ 
phenylmethylsulfonyl fluoride, and $12.5 \mathrm{mM} \mathrm{MgCl}$ ). The extract was aliquoted, frozen in liquid nitrogen, and stored at $-80^{\circ} \mathrm{C}$.

\section{Transcription Assays}

Transcription assays were performed as described by Manley et al. (1980) in a total volume of $25 \mu \mathrm{L}$ consisting of $15 \mu \mathrm{L}$ (100 to $150 \mu \mathrm{g}$ of protein) of the HNE, $400 \mu \mathrm{M}$ ATP, $400 \mu \mathrm{M}$ CTP, $400 \mu \mathrm{M}$ GTP, 50 $\mu \mathrm{M}{ }^{32} \mathrm{P}-\mathrm{UTP}(10 \mu \mathrm{Ci}, 3000 \mathrm{Ci} / \mathrm{mM})$, and $140 \mu \mathrm{M}$ EDTA. The reactions were initiated by the addition $(1 \mu \mathrm{g})$ of linearized single template. The reaction was incubated at $30^{\circ} \mathrm{C}$ for $1 \mathrm{hr}$ and was stopped by adding a buffer consisting of $8 \mathrm{M}$ urea, $0.5 \%$ SDS, $10 \mathrm{mM}$ EDTA, and $10 \mathrm{mM}$ Tris, pH 8.0. The samples were extracted twice with phenol-chloroform. The RNA was ethanol precipitated and applied to an $8 \%$ polyacrylamide-7 $\mathrm{M}$ urea sequencing gel. The gels were electrophoresed in $1 \times$ Tris-borate-EDTA buffer at $1400 \mathrm{~V}$ for 5 to $6 \mathrm{hr}$, dried, and then exposed to XRP film (Kodak).

Transcription competition was performed using double-stranded Em1b and mutant Em1b 24mer oligonucleotides (Genosys Biotechnologies, Inc., Woodlands, TX), and they are as follows: Em1b, 5'-GCGCTCGAGCGCTGCACACGTGCC-3' and 5'-CGCGTCGACGAGGCGGCACGTGTG-3'; and mutant Em1b, 5'-GCGCTCGAGCGCTGAATCTAGGCC-3' and 5'-CGCGTCGACGAGGCGGCCTAG-ATG-3' These oligonucleotides were annealed, filled in using the Klenow fragment of DNA polymerase I, and used to complete the G-box binding factor by adding excess molar concentrations (specified in the legend to Figure $4 \mathrm{C}$ ) at the same time as the template. All transcription reactions described were repeated at least three times.

\section{Immunodepletion of USF from the HNE}

Immunodepletion of USF from the HNE was conducted by the method of Luo et al. (1992) (Figures 8B to 8D). One hundred microliters of protein A-Sepharose beads (for fast flow; Pharmacia) was washed several times with $20 \mathrm{mM}$ Hepes and equilibrated with transcription dialysis buffer (mentioned above) and then packed into a 1 -mL pipette tip blocked with glass wool at the bottom. HNE $(150 \mu \mathrm{L})$ was incubated with the protein G-Sepharose-purified anti-USF ( 0.5 $\mathrm{mg}$ of protein per $\mathrm{mL}$ ) for $1 \mathrm{hr}$ with very gentle shaking on ice. This mixture of antibody and HNE was then passed over the protein A-Sepharose column prepared as given above. The control for this experiment was prepared in the same way but with the same amount of transcription dialysis buffer being added in place of the antibody. It was then passed through the column in the same manner.

Anti-USF was prepared in rabbits by using a $\beta$-galactosidase-sea urchin USF fusion protein. This antibody cross-reacts with human USF (Singh et al., 1994) and was a generous gift from M. Kozlowski (Massachusetts General Hospital, Charlestown, MA).

\section{Electrophoretic Mobility Shift Assays}

The electrophoretic mobility shift assay was performed in binding buffer consisting of $12 \mathrm{mM}$ Tris, $\mathrm{pH} 7.9,12 \%$ glycerol, $35 \mathrm{mM} \mathrm{KCl}$, $0.07 \mathrm{mM}$ EDTA, $1 \mathrm{mM}$ DTT, and $7.5 \mathrm{mM} \mathrm{MgCl}_{2}$ with 1 to $2 \mu \mathrm{g}$ of poly $(\mathrm{dl}-\mathrm{dC})$ as a nonspecific competitor, as indicated in the legend to Figure 5 . The HNE ( $5 \mu \mathrm{g}$ of protein) was added to 1 to $2 \mathrm{ng}$ of the labeled Em1b probe $(10,000$ to $15,000 \mathrm{cpm})$ and incubated for $15 \mathrm{~min}$ at room temperature. The Em1b oligonucleotide competitions were performed by using the Em1b and the Em1b mutant oligonucleotides mentioned above at the molar fold concentrations indicated in the appropriate figure legend. These competitor oligonucleotides were added to the reactions at the same time as the labeled probe. The reactions were resolved on a $4 \%$ nondenaturing gel (40:1 acrylamide to bis ratio) in $1 \times$ Tris-borate-EDTA. The gel was dried and then visualized by autoradiography.

\section{Preparation of Recombinant Proteins}

Recombinant MBP-EmBP1 (full length) and MBP-DVP1 (amino acids 190 to 692) were prepared as described by Hill et al. (1996), except for a few minor changes. After purification on the maltose column, these proteins were dialyzed for $1 \mathrm{hr}$ in transcription dialysis buffer $D$. The USF-purified recombinant protein was a gift from $P$. Pognonec (University of Nice, Nice, France).

\section{DNA Templates}

The plasmid pBM113 was described previously (Marcotte et al., 1988). This plasmid was linearized with EcoRV, resulting in two fragments of 5206 and $231 \mathrm{bp}$. The larger fragment was isolated from a TAE-agarose gel by dialysis and used as template 1 . The $5^{\prime}$ deletion series of the Em promoter (templates 2 to 5 ) was described by Marcotte et al. (1989). These deletions were treated in the same manner as described for template 1 . The maize rab28 plasmid, which contains the rab28 promoter fused to the $\beta$-glucuronidase (GUS) gene, was obtained from M. Pagès (Department de Genetica Molecular, Centro de Investigacion y Desarrollo, Barcelona, Spain), linearized with EcoRV, and treated in the same manner as the $\mathrm{Em}$ plasmids. The cauliflower mosaic virus (CaMV) $35 \mathrm{~S}$ promoter template was made by linearizing the PBM314 plasmid (Marcotte et al., 1988) with EcoRV. This plasmid contains the CaMV 35 S promoter inserted into the pBM113 plasmid in place of the Em promoter so that the template is identical to template 1 , except for the promoter region. The ubiquitin promoter (Christiansen et al., 1992) was in the form of plasmid pAHC27 (gift of P. Quail, U.S. Department of Agriculture Plant Gene Expression Center, Albany, CA), in which the ubiquitin promoter was cloned upstream of a GUS reporter gene in the PUC18 plasmid. It was linearized with EcoRV and used for transcription.

The dihydrofolate reductase (DHFR) template is the DHFR-chloramphenicol acetyltransferase template plasmid described by Swick et al. (1989). It consists of PUC18 containing the DHFR promoter region cloned upstream of the chloramphenicol acetyltransferase (CAT) reporter gene. The plasmid was linearized with Scal to generate a major transcript of 736 nucleotides and a minor transcript of 780 nucleotides. The adenovirus major late $(A d M L)$ template (AdML promoter cloned upstream of the CAT reporter gene) was also described by Swick et al. (1989). This plasmid was linearized with Ncol to generate a transcript of 642 nucleotides.

\section{Primer Extension}

Primer extension assay was performed according to the method of Sambrook et al. (1989), without any modifications. The primer used was $5^{\prime}$-GGTGCTCGACCGCTAAGGTT-3'. It was labeled with polynucleotide kinase and $\gamma^{32}$ P-ATP. Dioxynucleotide sequencing reactions (Sanger et al., 1977) were performed using this same primer. The labeled products of the primer extension and the sequencing re- 
actions were analyzed on an $8 \%$ denaturing gel, dried, and visualized by autoradiography.

\section{ACKNOWLEDGMENTS}

We thank Drs. M. Kozlowski and P. Pognonec for their generous gifts of USF antibodies and purified recombinant USF protein, and Drs. P. Quail and M. Pagès for the ubiquitin and rab28 promoters, respectively. We express our special thanks to our colleague Dr. Bill Marzluff (University of North Carolina, Chapel Hill) for some materials and especially for helpful comments and suggestions. This work was supported by a grant from the National Institutes of Health (No. GM 44288) to R.S.Q.

Received February 3, 1997; accepted August 21, 1997.

\section{REFERENCES}

Boller, T., and Wiekmen, A. (1986). Dynamics of vaduolar compartmentation. Annu. Rev. Plant Physiol. 37, 137-164.

Boston, R.S., and Larkins, B.A. (1986). Specific transcription of a 15-kilodalton zein gene in HeLa cell extracts. Plant Mol. Biol. 7, 771-779.

Chen, J.-L., Donatella, A., Verrijzer, C.P., Yokomori, K., and Tjian, R. (1994). Assembly of recombinant TFIID reveals differential coactivator requirements for distinct transcription activators. Cell 79, 93-105.

Christiansen, A., Sharrock, R.A., and Quail, P. (1992). Maize polyubiquitin genes: Structural, thermal perturbation of expression and transcript splicing, the promoter activity following transfer to protoplast by electrolysis. Plant Mol. Biol. 18, 675-689.

DeVos, K.M., Atkins, M.D., Chinoy, C.N., Guiltinan, M.G., Quatrano, R.S., and Gale, M.D. (1991). Chromosomal location and variability in wheat, barley and rye of a wheat gene encoding a bZIP (EmBP1). Theor. Appl. Genet. 82, 665-667.

Dignam, J.D., Lebovitz, R.M., and Roeder, R.G. (1983). Accurate transcription initiation by RNA polymerase II in a soluble extract from isolated mammalian nuclei. Nucleic Acids Res. 11, 1475-1489.

Ehrlich, K.C., Cary, J.W., and Ehrlich, M. (1992). A broad bean cDNA clone encoding a DNA-binding protein resembling mammalian CREB in its sequence specificity and DNA methylation sensitivity. Gene 117, 169-178.

Fan, H., and Sugiura, M. (1994). A plant basal in vitro system supporting accurate transcription of both RNA polymerase II- and IIIdependent genes: Supplement of green leaf component(s) drives accurate transcription of a light-responsive $r b c S$ gene. EMBO J. 14, 1024-1031.

Farnham, F.J., and Means, A.L. (1990). Sequences downstream of the transcription initiation site modulate the activity of the murine dihydrofolate reductase promoter. Mol. Cell. Biol. 12, 1390-1399.

Fischer, J.A., Giniger, E., Maniatis, T., and Ptashne, M. (1988). GAL4 activates transcription in Drosophila. Nature 332, 853-856.
Foley, R.C., Grossman, C., Ellis, J.G., Llewellyn, D.J., Dennis, E.S., Peacock, W.J., and Singh, K:B. (1993). Isolation of a maize bZIP protein subfamily: Candidates for the ocs-element transcription factor. Plant J. 3, 669-679.

Foster, R., Izawa, T., and (Chua, N.:H. $^{-}$(1994). Plant bZIP proteins gather at ACGT elements. FASEB.J.:8, 192-200.

Frohnmeyer, H., Hahlbrock, .K., and Schafer, E. (1994). A lightresponsive in vitro transcription system from evacuolated parsley protoplasts. Plant J. 5, 437-449.

Giuliano, G., Pichersky, E., Malik, V.S., Timko, M.P., Scolnik, P.A., and Cashmore, A.R. (1988). An evolutionarily conserved protein binding sequence of a plant light-regulated gene. Proc. Natl. Acad. Sci. USA 85, 7089-7093.

Guiltinan, M.J., Marcotte, W.R., Jr., and Quatrano, R.S. (1990). A plant leucine zipper protein that recognizes an abscisic acid response element. Science 250, 267-271.

Haas, M., and Feix, G. (1992). Two different cDNAs encoding TFIID proteins of maize. FEBS Lett. 301, 294-298.

Hattori, T., Terada, T., and Terada-Hamasuna, S. (1994). Sequence and functional analyses of the rice gene homologous to the maize Vp1. Plant Mol. Biol. 24, 805-810.

Hattori, T., Terada, T., and Hamasuna, S. (1995). Regulation of the Osem gene by abscisic acid and the transcriptional activator VP1: Analysis of cis-acting promoter elements required for regulation by abscisic acid and VP1. Plant J. 7, 913-925.

Hill, A., Nantel, A., Rock, C.D., and Quatrano, R.S. (1996). The product of the Viviparous-1 gene enhances the DNA-binding activity of several transcription factors. J. Biol. Chem. 271, 3366-3374.

Joshi, C.P. (1987). An inspection of the domain between putative TATA box and translation start sites in 79 plants. Nucleic Acids Res. 15, 6643-6653.

Kakidani, H., and Ptashne, M. (1988). GAL4 activates gene expression in mammalian cells. Cell 52, 161-167.

Katagiri, F., Lam, E., and Chua, N.-H. (1989). Two tobacco DNAbinding proteins with homology to the nuclear factor CREB. Nature 340, 727-730.

Katagiri, F., Yamazaki, K.-I., Horikoshi, M., Roeder, R.G., and Chua, N.-H. (1990). A plant DNA-binding protein increases the number of active preinitiation complexes in a human in vitro transcription system. Genes Dev. 4, 1899-1909.

Katagiri, F., Seipel, K., and Chua, N.-H. (1992). Identification of a novel dimer stabilization region in a plant bZIP transcription activator. Mol. Cell. Biol. 12, 4809-4816.

Kawata, T., Imada, T., Shiraishi, H., Okada, K., Shimura, Y., and Iwabushi, M. (1992). A cDNA clone encoding HBP-1b homologue in Arabidopsis thaliana. Nucleic Acids Res. 20, 1141.

Knight, C.D., Sengal, A., Atwal, K., Wallace, J.C., Cove, D.J., Coates, D., Quatrano, R.S., Bahadur, S., Stockley, P.G., and Cumming, A.C. (1995). Molecular responses to abscisic acid and stress are conserved between moss and cereal. Plant Cell 7, 499-506.

Kozak, M. (1984). Compilation and analysis of sequences upstream from the transcriptional start site in eukaryotic mRNA. Nucleic Acids Res. 12, 857-872. 
Li, J.-M., Parson, R.A., and Marzluff, W.F. (1994). Transcription of the sea urchin $U 6$ gene in vitro requires a TATA-like box, a proximal sequence element, and sea urchin USF, which binds an essential E box. Mol. Cell. Biol. 14, 2191-2200.

Litts, J.C., Colwell, G.W., Chakerian, R.L., and Quatrano, R.S. (1991). Sequence analysis of a functional member of the $E m$ gene family from wheat. DNA Sequence 1, 263-274.

Luo, Y., Fuji,T., Gerster, T., and Roeder, R.G. (1992). A novel B cell-derived coactivator potentiates the activation of immunoglobulin promoters by octamer-binding transcription factors. Cell 71, 231-241.

Ma, J., Prizibilla, E., Hu, L., Bogorad, L., and Ptashne, M. (1988). Yeast activators stimulate plant gene expression. Nature 334, 631-633.

Manley, J.L., Fire, A., Cano, A., Sharp, P.A., and Gefter, M.L. (1980). DNA-dependent transcription of adenovirus genes in a soluble whole-cell extract. Proc. Natl. Acad. Sci. USA 77, 3855-3859.

Marcotte, W.R., Jr., Bayley, C.C., and Quatrano, R.S. (1988). Reg ulation of a wheat promoter by abscisic acid in rice protoplasts. Nature 335, 454-457.

Marcotte, W.R., Jr., Russell, S.H., and Quatrano, R.S. (1989). Abscisic acid-responsive sequences from the $E m$ gene of wheat. Plant Cell 1, 969-976.

McCarty, D.R., Hattori, T., Carson, C.B., Vasil, V., Lazar, M., and Vasil, I.K. (1991). The viviparous-1 developmental gene of maize encodes a novel transcriptional activator. Cell 66, 895-905.

McKendree, W.L., and Ferl, R.J. (1992). Functional elements of the Arabidopsis Adh promoter include the G-box. Plant Mol. Biol. 19, 859-862.

Mukumoto, F., Hirose, S., Imaseki, H., and Yamazaki, K.I. (1993). DNA sequence requirement of a TATA element-binding protein from Arabidopsis for transcription in vitro. Plant Mol. Biol. 23, 995-1003.

Nantel, A., and Quatrano, R.S. (1996). Characterization of three rice bZIP factors, including two inhibitors of EmBP-1 DNA binding activity. J. Biol. Chem. 271, 31296-31305.

Parcy, F., Valon, C., Raynal, M., Gaubier-Comella, P., Delseny, M., and Giraudat, J. (1994). Regulation of gene expression programs during Arabidopsis seed development: Roles of the $A B / 3$ locus and of endogenous abscisic acid. Plant Cell 6, 1567-1582.

Pla, M., Gómez, J., Goday, A., and Pagès, M. (1991). Regulation of the abscisic acid-responsive gene rab28 in maize viviparous-1 mutants. Mol. Gen. Genet. 230, 394-400.

Pla, M., Vilardell, J., Guiltinan, M.J., Marcotte, W.R., Niogret, M.F., Quatrano, R.S., and Pagès, M. (1993). The cis-regulatory element CCACGTGG is involved in $A B A$ and water-stress responses of the maize gene rab28. Plant Mol. Biol. 21, 259-266.

Pysh, L.D., Aukerman, M.J., and Schmidt, R.J. (1993). OHP1: A maize basic domain/leucine zipper protein that interacts with Opaque2. Plant Cell 5, 227-236.

Rock, C.R., and Quatrano, R.S. (1995). The role of hormones during seed development. In Plant Hormones: Physiology, Biochemistry and Molecular Biology, 2nd ed, P.J. Davies, ed (Dordrecht, The Netherlands: Kluwer Academic Publishers), pp. 671-697.

Roy, A.L., Meristerenst, M., Pognonec, P., and Roeder, R.G. (1991). Cooperative interaction of an initiator-binding transcription initiation factor and the helix-hoop-helix activator USF. Nature $354,245-248$.

Sambrook, J., Fritsch, E.F., and Maniatis, T. (1989). Molecular Cloning: A Laboratory Manual, 2nd ed. (Cold Spring Harbor, NY: Cold Spring Harbor Laboratory Press).

Sanger, F., Nicklen, S., and Coulson, A.R. (1977). DNA sequencing with chain-terminating inhibitors. Proc. Natl. Acad. Sci. USA 74, 5463-5467.

Sawadogo, M., and Roeder, R.G. (1985). Interaction of a gene-specific transcription factor with the adenovirus major late promoter upstream of the TATA box region. Cell 43, 165-175.

Schilling, L.J., and Farnham, P.J. (1995). The bidirectionally transcribed dihydrofolate reductase and rep-3a promoters are growth regulated by distinct mechanisms. Cell Growth Differ. 6, 541-548.

Schindler, U., Beckmann, H., and Cashmore, A.R. (1992a). TGA1 and $\mathrm{G}$-box binding factors: Two distinct classes of Arabidopsis leucine zipper proteins compete for the $G$-box-like element TGACGTGG. Plant Cell 4, 1309-1319.

Schindler, U., Terzaghi, W., Beckmann, H., Kadesch, T., and Cashmore, A.R. (1992b). DNA binding site preference and transcriptional activation properties of the Arabidopsis transcription factor GBF1. EMBO J. 11, 1275-1289.

Schweizer, P., and Mosinger, E. (1994). Initiator-dependent transcription in vitro by a wheat germ chromatin extract. Plant Mol. Biol. 25, 115-130.

Singh, S.S., Luo, Z., Kozlowski, M.T., and Erlichman, J. (1994). Association of USF and c-Myc with a helix-loop-helix consensus motif in the core promoter of the murine type IIB regulatory subunit gene of a cyclic adenosine $3^{\prime}, 5^{\prime}$-monophosphate-dependent protein kinase. Mol. Endocrinol. 8, 1163-1174.

Sugiura, M. (1996). Plant in vitro transcription: The opening of a new era. Trends Plant Sci. 1, 41.

Suzuki, M., Chien, Y.K., and McCarty, D.R. (1997). The conserved B3 domain of VIVIPAROUS1 has a cooperative DNA binding activity. Plant Cell 9, 799-807.

Swick, A.G., Blake, M.C., Kahn, J.W., and Azizkhan, J.C. (1989). Functional analysis of $\mathrm{GC}$ element binding and transcription in the hamster dihydrofolate reductase gene promoter. Nucleic Acids Res. 17, 9291-9304.

Timchenko, N., Wilson, D.R., Taylor, L.R., Abdelsayed, S., Wilde, M., Sawadogo, M., and Darlington, G.J. (1995). Autoregulation of the human C/EBPa gene by stimulation of upstream stimulatory factor binding. Mol. Cell. Biol. 15, 1192-1202.

Vasil, V., Marcotte, W.R., Jr., Rosenkrans, L., Cocciolone, S., Vasil, I.K., Quatrano, R.S., and McCarty, D.R. (1995). Overlap of Viviparous1 (VP1) and abscisic acid response elements in the Em promoter: G-box elements are sufficient but not necessary for VP1 transactivation. Plant Cell 7, 1511-1518.

Weisshaar, B., Armstrong, G.A., Block, A., da Costa e Silva, O., and Hahlbrock, K. (1991). Light-inducible and constitutively expressed DNA-binding proteins recognizing a plant promoter element with functional relevance in light responsiveness. EMBO J. 10, 1777-1786.

Williamson, J.D., and Scandalios, J.G. (1992). Differential response of maize catalases to abscisic acid: VP1 transcriptional activator is not required for abscisic acid-regulated Cat1 expression. Proc. Natl. Acad. Sci. USA 89, 8842-8846. 
Yamaguchi, Y., Mukumoto, F., Imaseki, H., and Yamazaki, K.-I. (1994). Preparation of an in vitro transcription system of plant origin with methods and templates for assessing its fidelity. In Plant Molecular Biology Manual E2, S.B. Gelvin and R.A. Schilperoort, eds (Dordrecht, The Netherlands: Kluwer Academic Publishers), pp. 1-15.

Yamazaki, K.-I., Katagiri, F., Imaseki, H., and Chua, N.-H. (1990). TGA1a, a tobacco DNA-binding protein, increases the rate of initi- ation in a plant in vitro transcription system. Proc. Natl. Acad. Sci. USA 87, 7035-7039.

Zhang, B., Foley, R.C., and Singh, K.B. (1993). Isolation and characterization of two related Arabidopsis ocs-element bZIP binding proteins. Plant J. 4, 711-716.

Zhu, Q., Dabi, T., and Lamb, C. (1995). TATA box and initiator functions in accurate transcription of a plant minimal promoter in vitro. Plant Cell 7, 1681-1689. 
Effect of the nuclear factors EmBP1 and viviparous1 on the transcription of the Em gene in HeLa nuclear extracts.

M A Razik and R S Quatrano

Plant Cell 1997;9;1791-1803

DOI 10.1105/tpc.9.10.1791

This information is current as of July 22, 2020

Permissions

eTOCs

CiteTrack Alerts

Subscription Information
https://www.copyright.com/ccc/openurl.do?sid=pd_hw1532298X\&issn=1532298X\&WT.mc_id=pd_hw1532 $298 X$

Sign up for eTOCs at:

http://www.plantcell.org/cgi/alerts/ctmain

Sign up for CiteTrack Alerts at:

http://www.plantcell.org/cgi/alerts/ctmain

Subscription Information for The Plant Cell and Plant Physiology is available at: http://www.aspb.org/publications/subscriptions.cfm 